\title{
HYDROTHEMAL AGEING OF GRAPHENE/CARBON NANOTUBES/EPOXY HYBRID NANOCOMPOSITES
}

\author{
C. ARRIBAS ${ }^{1}$, M.G. PROLONGO ${ }^{1 *}$, M. SÁNCHEZ-CABEZUDO ${ }^{1}$, R. MORICHE ${ }^{2}$, \\ S.G.PROLONGO ${ }^{2}$
}

1) Material Science and Engineering Area. ESCET. Universidad Rey Juan Carlos, c/Tulipán s/n, Móstoles, 28933, Madrid, Spain.

2) Dpt. Aerospace Materials and Production. E.T.S.I. Aeronáutica y del Espacio. Universidad Politécnica de Madrid. Spain.

* corresponding author: mg.prolongo@upm.es 


\section{ABSTRACT}

The hydrothermal ageing of hybrid nanocomposites formed by carbon nanotubes (CNTs) and graphene nanoplatelets (GNPs) incorporated into an epoxy matrix was studied. In order to avoid the weak interface, amine functionalized GNPs were used which are reactive with the epoxy matrix. The content of CNTs was $0.1 \mathrm{wt} \%$ and the GNPs content ranged from 5 to $12 \mathrm{wt} \%$. The isothermal water absorption was measured until very long times (almost two years). Reduction of water absorption with the addition of either carbon nanotubes or graphene nanoplatelets has been found independently of the ageing time. The results can be described by a two-stage diffusion model with the first and second stages being diffusion and matrix relaxation controlled respectively. The obtained diffusion coefficient for the neat epoxy thermoset is higher than those of nanocomposites, which decreases with the increase of GNPs content. The effect of water absorbed on the dynamic-mechanical properties and on the electrical conductivity of the nanocomposites was analyzed. Whatever the temperature the storage modulus shows a continuous increase with the increase of the nanoparticles content, the effect of hydrothermal ageing is to decrease the modulus only in the glassy state ( $T<$ $T_{g}$ ). Water aged specimens show broadening of the $\tan \delta$ peak related to the plasticization of the matrix. $T_{g}$ of aged specimens coincides with the one of the unaged matrix, indicating that hydrothermal ageing does not produce noticeable matrix damage even after two years. Water aged specimens show similar electrical conductivity than unaged ones, which is another indication of no degradation of samples by water.

Key words: epoxy, graphene, carbon nanotubes, water ageing, diffusion coefficient, two stage diffusion, thermo-mechanical and electrical properties. 


\section{Introduction}

Nanocomposites formed by polymer matrices with graphitic nanofillers are a new class of materials with interesting properties. Carbon nanotubes (CNTs) and graphene nanoplatelets (GNPs) are one and two dimensional nanofillers that are used in polymer nanocomposites to improve mechanical properties, thermal and electrical conductivity among other properties [1-4]. Graphene nanoplatelets also provide low permeability, being available at relatively low cost compared to CNTs. On the other hand, carbon nanotubes dispersed in polymer matrices achieve electrical conductivity percolation threshold at very low concentrations.

Few works have been carried out to investigate the effects of combining CNTs and GNPs as fillers of epoxy matrices, although the combination of GNPs and CNTs in a polymer matrix to form hybrid nanocomposites offers the opportunity to obtain new materials. Making hybrid nanocomposites is aimed to reach synergistic effects between GNPs and CNTs. Most of the research published works on epoxy hybrid nanocomposites refer increment of mechanical properties [1, 4-9] and some of them show a synergic increase by the joint addition of CNTs and GNPs [1, 6-8]. The improvement of properties is attributed to the formation of an interconnected network in which the carbon nanotubes create bridges between the graphene nanosheets [1,5]. Electrical properties of CNTs and GNPs hybrid epoxy nanocomposites are scarcely reported [4,6,10-13]. The changes in electrical conductivity by the simultaneously addition of CNTs and GNPs, is related to the CNTs bridging between adjacent GNPs which results in a more efficient conductive network $[4,11,13]$. Moreover thermal conductivity also gives a synergistic behavior, but electrical properties seem to remain unaffected or even decrease [6].

Chemical modification of carbon nanofillers has been done to achieve a strong adhesion at the nanofiller/polymer interface [14-16]. Weak interfaces with the matrix are more critical for GNPs than for CNTs, causing a higher decrease of the mechanical properties of composites [17,18]. However chemical modification undesirably affects the conductivity of the nanocomposites $[6,19,20]$.

When materials are exposed to environmental agents, such as temperature and moisture, modification in their properties can occur. The ageing processes are related to physical and chemical changes, that need to be studied to evaluate the material durability, that is the time up to which the properties changes are acceptable. Moreover it is interesting to 
know if these changes are reversible. Epoxy thermosets are widely used in the industry due to their superior characteristics such as good mechanical properties. However, it is known that hydrothermal ageing of epoxy thermosets may cause modification of glass transition temperature (plasticization), changes in cross-linking, and even chemical degradation [21-27]. Epoxy composites are exposed to a humid environment in many applications. The water uptake of polymers can be reduced by incorporating graphene nanoplatelets [17, 28-30].The diffusion of water in epoxy graphene nanocomposites decreases in relation to neat epoxy that is explained by the more tortuous pathways that water molecules have to take to penetrate in the nanocomposite. However, it should be considered that the exposure to water under long periods can affect the thermomechanical properties of the nanocomposites. In relation to water absorption the combined effect of the addition of CNTs and GNPs into epoxy resins, has scarcely been studied [8]

The aim of this work is to study the water uptake of hybrid epoxy nanocomposites doped with CNTs and GNPs reaching long absorption times (almost 2 years). Moreover it is investigated how the hydrothermal ageing at long times influences the mechanical and electrical properties of the nanocomposites. Amine functionalized graphene nanoplatelets were selected in order to enhance the interface between the nanofiller and matrix. The studied epoxy hybrid nanocomposites contained $0.1 \mathrm{wt} \%$ of CNTs which gives percolation referred to electrical conductivity and different amounts of GNPs (5$12 \mathrm{wt} \%)$. In a previous work we have reported that these nanocomposites present a synergic effect on the stiffness [6]. The results here obtained will be helpful in understanding the effects of hydrothermal ageing on the long term durability of epoxy hybrid nanocomposites.

\section{Experimental}

\subsection{Materials}

The epoxy resin Araldite LY556 (diglycidyl ether of bisphenol A type (DGEBA)) was cured with Araldite XB3473 (aromatic diamine, mainly diethyltoluenediamine) both were purchased from Huntsman. Functionalized graphene nanoplatelets, with a thickness lower than $4 \mathrm{~nm}$, and $5 \mu \mathrm{m}$ of lateral size, were supplied by Cheaptubes. They were amino-functionalized (0.5 wt\%) and their specific area was $750 \mathrm{~m}^{2} / \mathrm{g}$. Carbon 
nanotubes NC7000 were purchased from Nanocyl, with length lower than $1.5 \mu \mathrm{m}$ and average diameter $9.5 \mathrm{~nm}$ and their specific area was $250-300 \mathrm{~m}^{2} / \mathrm{g}$.

\subsection{Sample preparation}

The mixtures LY+XB were prepared in stoichiometric ratio (LY556:XB3473 = 100:23 w/w). Table 1 collects the composition of the studied samples. The percentages of nanofiller were selected considering the electrical percolation threshold [5]: the electrical percolation with CNTs (NC7000) is close to 0.1 wt \%, while the threshold with amino-functionalized GNPs is higher than $5 \mathrm{wt} \%$. Appropriate amounts of CNTs and GNPs were loaded into the epoxy resin to obtain hybrid samples. The dispersion of GNPs and CNTs into epoxy resin (LY) was carried out by mini-calandering (three-roll miller Exakt 80E GmbH). The rolling speed was $250 \mathrm{rpm}$ and the gap size between adjacent rolls was $5 \mu \mathrm{m}$. The calandering process was applied four consecutive times. Afterwards, the dispersions were degassed under vacuum at $80{ }^{\circ} \mathrm{C}$ for $15 \mathrm{~min}$. Then, the hardener was added at $80{ }^{\circ} \mathrm{C}$. These dispersions were cast onto aluminum open molds that were treated with a mold release product. The curing consisted of heating at $140{ }^{\circ} \mathrm{C}$ for $8 \mathrm{~h}$. The cured samples were slowly cooled to room temperature.

\subsection{Measurements}

Hydrothermal aging of the specimens has been carried out by immersion in distilled water at $40{ }^{\circ} \mathrm{C}$ controlled to better than $\pm 0.1{ }^{\circ} \mathrm{C}$. Two specimens have been tested for each composition. The specimens have dimensions of 45x10x1.9 $\mathrm{mm}^{3}$. The specimens were periodically removed from water, carefully wiped to remove excess of water and weighted with accuracy of $\pm 0.01 \mathrm{mg}$. The change in weight of the specimens was monitored as a function of the immersion time, $t$. The total period for which the sample was out of water was less than 1 minute. The water sorption, that is, the water uptake at time $t$, was calculated as:

$$
M_{t}=\left(\Delta W / W_{0}\right)
$$

being $W_{0}$ the initial weight of the dry sample and $\Delta W=W_{t}-W_{0}$, where $W_{t}$ is the weight of the hydrothermally aged specimen at each time.

Dynamic mechanical thermal analysis (DMTA) was performed in dual cantilever bending mode using a DMTA V Rheometric Scientific instrument. Measurements were done at $1 \mathrm{~Hz}$, with temperature increasing from $30{ }^{\circ} \mathrm{C}$ to $220^{\circ} \mathrm{C}$ at a heating rate of 2 
${ }^{\circ} \mathrm{C} \cdot \mathrm{min}^{-1}$. Specimen dimensions were: $45 \times 10 \times 1.9 \mathrm{~mm}^{3}$. Two samples were measured per each composition. The elastic or storage modulus ( $\left.E^{\prime}\right)$, the loss modulus ( $\left.E^{\prime \prime}\right)$ and loss tangent $(\tan \delta)$ were recorded for each frequency as a function of temperature. The maxima in $\tan \delta$-temperature curves were determined to identify the $\alpha$-relaxations associated to the glass transitions.

DC volume electrical conductivity of nanocomposites was measured according to ASTM D257 using a Source Meter Unit instrument (KEITHLEY 2410). The applied voltage was within the range of $0-15 \mathrm{~V}$. Two samples were measured per each composition. Specimen dimensions were: 10x10x1 $\mathrm{mm}^{3}$. Silver paint was used to minimize the electrical contact resistance between the samples and the copper electrodes used as contacts. Aged samples were taken out of the water immediately before their measurement: they were gently wiped out with a tissue and afterward the silver paint was applied and they were measured immediately. The applied voltage was within the range of $0-15 \mathrm{~V}$ in 5 steps. The electrical conductivity was then calculated from the characteristic I-V curve by using the Ohm's Law. Two samples were measured per each composition. Specimen dimensions were: 10x10x1 $\mathrm{mm}^{3}$.

Nanocomposites morphology was analyzed by Field Emission Gun Electron Scanning Microscopy (FEG-SEM, Nova NanoSEM Fei. Houston. USA). The samples were coated with a thin layer ( $2 \mathrm{~nm})$ of Pt.

\section{Results and discussion}

\subsection{Morphological characterization}

The SEM micrographs of fracture surfaces of unaged and water aged 8G-0.1C nanocomposite are shown in Fig. 1. The morphological characterization of similar samples was investigated in a previous work [6]. It can be summarized that there were not detected aggregates of CNTs or GNPs in the samples. On hybrid samples CNTs tend to selectively locate between GNPs forming bridges between nanoplatelets. Micrographs of unaged samples are shown in Fig. 1: at low magnification $1 \mathrm{~A}$ and at high magnifications $1 \mathrm{~B}$ and $1 \mathrm{C}$. The micrograph $1 \mathrm{~B}$ shows mainly a graphene nanoplatelet and its interface with the matrix while micrograph $1 \mathrm{C}$ shows a region with high concentration of CNTs. After hydrothermal ageing: micrographs $2 \mathrm{~A}$ at low magnification and $2 \mathrm{~B}$ and $2 \mathrm{C}$ at high magnifications are also shown in Fig. 1. The fractography of the samples can be observed in micrographs at lower magnification (1A 
and $2 \mathrm{~A}$ ). It seems that the fracture, after the immersion in water (2A), is rougher with different fracture planes while the surface of the unaged sample (1A) seems to be smoother. This is explained because the aged sample is plasticized by absorbed water, showing higher plastic deformation and therefore higher toughness. At high magnification, the details of the interface between the graphene nanoplatelet and the epoxy matrix can be observed in the micrographs $1 \mathrm{~B}$ and $2 \mathrm{~B}$. It seems that the interface of the non-aged sample is strong and continuous, without showing loss of adherence (marked with white rectangles in micrograph 1B). However, after the hydrothermal ageing, some spaces are detected at the interfaces between the graphene and the matrix (marked with white rectangles in micrograph 2B). As it is well known, this lack of adherence could be associated with the absorption of water that weakens the interfaces in spite of the chemical bounding between amine modified GNPs and the epoxy matrix. Finally, the micrographs $1 \mathrm{C}$ and $2 \mathrm{C}$ show the dispersed nanotubes in the matrix. The length of the pulled out nanotubes is slightly lower in the unaged sample (1C) regard to the water aged sample (2C). Again, this implies that the CNT-epoxy interface was sligthtly stronger before water absorption. Even though water seems to weaken the interface the micrographs after water ageing do not show the development of microvoids, indicating that almost no damage has been produced in the hybrid epoxy nanocomposites.

\subsection{Water absorption of epoxy nanocomposites}

The water absorption curves ( $M_{t}$ versus $t^{1 / 2} \cdot h^{-1}$, being $h$ the specimen thickness), for Epoxy, 0.1C, and 12G-0.1C samples are plotted in Fig. 2. Two specimens were tested for each composition and both followed the same pattern. The other compositions (12G, 5G-0.1C, 8G-0.1C and 10G-0.1C) present similar behaviour. The curves are linear in the early stage of absorption, which is one of the characteristics of fickian diffusion [31]. After the initial rapid water uptake, a continuing absorption of water takes place at very slow rate. It can be seen that even after long times of immersion ( 16000 h which correspond to $\sim 4000 \mathrm{~s}^{1 / 2} \cdot \mathrm{mm}^{-1}$ ) equilibrium has not been reached and the samples continued absorbing water at very low rate. Diamine cured epoxy thermosets absorb water due to the presence of polar groups, mainly hydroxyl groups generated by the epoxy-amine reaction. It is worth to note that the water absorbed by this epoxy thermoset is small compared to other epoxy systems like DGEBA cured with diamine 
diphenyl methane-DDM [32] reaching $~ 1.74 \mathrm{wt} \%$ and $~ 3 \mathrm{wt} \%$ respectively for 2000 $\mathrm{s}^{1 / 2} \cdot \mathrm{mm}^{-1}$ under similar conditions. The more compact polymer structure, the smaller its free volume is [33-34] and there is less space available for water absorption therefore this epoxy thermoset (LY-XB) behaves as a tighter network than DGEBA-DDM.

As shown in Fig. 2, the amount of absorbed water is reduced by the addition of carbon nanofillers. In order to compare the effect of the different carbon nanofillers Fig. 3 shows the variation of $M_{t}$ corresponding to $2500 \mathrm{~s}^{1 / 2} \cdot \mathrm{mm}^{-1}$ with the content of GNPs. As expected $M_{t}$ of the hybrid nanocomposites, decreases with increasing GNPs content, and the dependence with the GNPs content is almost linear. Obviously, the matrix is the nanocomposite's component accountable of the water sorption since graphene nanoplatelets and carbon nanotubes hardly absorb water. Therefore, the water uptake referred to the epoxy mass in the specimen, $M_{t}^{e p}$, would be more adequate in order to compare the capability of water absorption of the matrix in the nanocomposites. The values of $M_{t}^{e p}$ (calculated taking $W_{0}$ in Eq. (1) as the mass of epoxy matrix in the specimen) are also plotted in Fig.3. As expected for the 0.1C nanocomposite, $M_{t}^{e p}$ is similar to $M_{t}$ due to the low content of CNTs, while for nanocomposites containing graphene nanoplatelets $M_{t}^{e p}$ is higher than $M_{t}$. It can be observed that $M_{t}$ of neat epoxy thermoset is slightly higher than $M_{t}^{e p}$ of nanocomposites this could be justified by the increase of hydrophobicity of the samples containing GNPs or CNTs [17]. The addition of CNTs lowers the water absorption of neat epoxy and hybrid nanocomposite, being this effect higher for the neat epoxy because the hydrophobicity change is higher respect to the sample that contains GNPs.

Reduction of water absorption with the addition of carbon nanofillers has been explained in terms of hindered intermolecular movements of the epoxy due to the fillers [28]. Indeed, in similar samples to the ones here studied [6] it was found that the presence of the nanofillers leads to increase the $T_{g}$. Consequently, the nanocomposites have lower free volume than neat epoxy and the lower is the level of absorbed water compared to the neat epoxy.

In Fig 3 it can be seen that $M_{t}^{e p}$ slightly increases as the graphene content increases this can be explained taking into account that amine groups of GNPs can modify the stoichiometry of the epoxy matrix lowering its crosslinking degree, thus increasing free volume, this effect would be proportional to the GNPs content. Therefore there are contrary effects, on one hand the water absorption decreases due to the carbon nanofillers hydrophobicity and to the epoxy restricted mobility around them, on the 
other hand water sorption increases due to the lower crosslinking degree induced by stoichiometry unbalances.

Additionally, it has been reported that the degradation of the graphene-epoxy interface, can originated voids where the water can be trapped [17]. However, as it was above discussed, in the systems here studied no voids or damage were detected, it was only observed weakening of interfaces that facilitates to pull out the fillers.

\subsection{Diffusion coefficients}

Fickian behavior assumes that the water concentration gradient is the driving force for diffusion. Therefore, the absorption curves are linear at the initial stage and they asymptotically approach the equilibrium water absorption, $M_{\infty}$, and the diffusion coefficients can be calculated according to Fick’s second law [32]:

$$
M_{t}=\left(1-\exp \left[-7.3\left(\frac{D t}{h^{2}}\right)^{0.75}\right]\right) M_{\infty}
$$

where $D$ is the diffusion coefficient, $h$ is the thickness of the specimen, and $M_{\infty}$ is the equilibrium value of the water uptake.

The water absorption curves shown in Fig. 2, reveals diffusion behavior in two steps. The absorption at the early times associated to fickian diffusion and after that, a continuous increase of absorption. This behavior has been observed in other systems [23, 32, 35-39]. In such cases, a two-stage diffusion model can be used to fit the data: the first stage is controlled by a concentration gradient, according to Fick's law and the second one takes into account the steady increment of the absorption:

$$
M_{t}=(1+k \sqrt{t})\left(1-\exp \left[-7.3\left(\frac{D \cdot t}{h^{2}}\right)^{0.75}\right]\right) M_{\infty 0}
$$

where $M_{\infty 0}$ is the quasi-equilibrium uptake of the diffusion-dominated first stage and $D$ is the diffusion coefficient. The term $\left(1+k t^{1 / 2}\right)$ characterizes the second stage, $k$ is a constant that represents the rate of absorption of the second stage and it has been proposed to be related to the rate of relaxation of the polymeric matrix [35]. The structural relaxation is assumed to be much slower than the diffusion process. This is particularly true in the systems studied here, in which the aging temperature $\left(40^{\circ} \mathrm{C}\right)$ is much lower than the $T_{g}$ of the systems (around $170^{\circ} \mathrm{C}$ ). 
The experimental data $M_{t}$ versus $t^{1} / 2 \cdot h^{-1}$ were fitted using Eq. (3). In all the cases a good agreement between the experimental data and fitted results was obtained as it is illustrated in Fig. 2. The obtained values for $M_{\infty 0}, D$ and $k$ are listed in Table 2. As all specimens have similar dimensions, the "edge effect" correction [32] on $D$ is similar in all them. The values of $M_{\infty 0}$ are lower than the values of $M_{t}$ corresponding to 2500 $\mathrm{s}^{1 / 2} \cdot \mathrm{mm}^{-1}$ though the variation with nanofiller content follow the same trend. The addition of any of the nanofiller produces a reduction on the diffusion coefficient. The reduction of $D$ by the addition of GNPs seems to be proportional to the GNPs content as it is illustrated in Fig. 4. The graphene nanoplatelets force water to follow a tortuous pathway leading to the decrease of $D$. The addition of $0.1 \%$ CNTs to neat epoxy thermoset and to $12 \mathrm{G}$ nanocomposite has a similar effect lowering $D$.

Regarding the second stage of absorption the $k$ values of $0.1 \mathrm{C}$ nanocomposite and Epoxy are similar while for GNPs nanocomposites $k$ values are lower (see Table 2). This effect can be related to the anchorage of the amino functionalized GNPs to the epoxy matrix that constrains the segmental motions of the epoxy matrix and makes slower the epoxy relaxation. Therefore the amino functionalized GNPs which react with the matrix originate strong interfaces being water diffusivity and the level of water uptake lower than neat epoxy thermoset. The behavior of the nanocomposites is in accordance with their morphology, that is, agglomerates and voids that would increase diffusivity [40] were not detected.

The $T_{g}$ values (see Table 3 ) are much higher than the hydrothermal ageing temperature $\left(40^{\circ} \mathrm{C}\right)$ so the epoxy relaxations should occur very slowly, accordingly a constant value for $M_{t}$ was not reached even after16000 hours of hydrothermal ageing. In less tightly crosslinked epoxies having lower $T_{g}$ (around $100^{\circ} \mathrm{C}$ ) [23] the two stage absorption curves disappears at water aging temperature $\left(70^{\circ} \mathrm{C}\right)$ near the $T_{g}$ of the network, where the relaxation takes place at shorter times.

\subsection{Effect of hydrothermal ageing on the thermo-mechanical properties}

To evaluate the effects of hydrothermal ageing on the thermo-mechanical properties DMTA tests were performed for: “as-produced” and "water-aged” specimens (after 1 and almost 2 years of immersion in water at $40^{\circ} \mathrm{C}$ ). Fig. 5 shows $\tan \delta$-temperature curves for non-aged and water aged specimens. The $\tan \delta$ curve of the nonhydrothermally aged samples shows one relaxation peak at high temperature, located 
around $170^{\circ} \mathrm{C}$, with narrow distribution indicating that the epoxy network behaves as homogeneous. The water uptake influences the height and shape of $\tan \delta$ curves, and water aged specimens show widening of the $\tan \delta$ peaks. Aged neat epoxy and $0.1 \mathrm{CNT}$ nanocomposite show an asymmetric broadening of the $\tan \delta$ peak. While for the samples containing GNPS a shoulder is observed at lower temperatures. The peak is located around $170^{\circ} \mathrm{C}$ and the shoulder around $145^{\circ} \mathrm{C}$. It is worth to note that as the peak broadens the height of the peak lowers. The breadth (area) of the $\tan \delta$ peak is related to the damping behavior and the peak width represents the temperature range over which the glass transition takes place. Thus, a broad $\tan \delta$ peak can be attributed to a more heterogeneous network containing different relaxations regions.

The values of temperature for the maximum in $\tan \delta$ curves ( $T \tan \delta_{\max }$ ) for unaged and aged samples ( 1 and 2 years of hydrothermal ageing) are reported in Table 3. It can be seen that for unaged samples, the $T_{g}$ of nanocomposites is higher than the $T_{g}$ of neat epoxy. The presence of CNTs and amino functionalized GNPs in the epoxy matrix induces a noticeable increase in $T_{g}$ associated to the hindrance of polymer chainsegments movements [6]. The values reported in Table 3 show that the $\tan \delta$ peak appears at similar temperatures in aged and unaged specimens, which evidences that no chemical degradation took place after two years of water immersion. On the other hand, the DMTA scans after one and two years of hydrothermal ageing are similar indicating that the absorption of water during the second year does not affect the matrix, which is in accordance with the small amount of absorbed water during the second ageing year. Therefore the absorbed water did not produce noticeable matrix damage even during large periods of ageing.

It has been frequently reported spliting of $\tan \delta$ peaks for hydrothermally aged polymers and attributed either to drying effect during DMTA tests or to the heterogeneous plasticization of epoxy matrix by the absorbed water [32,39,41-43]. Whatever the origin, the shoulder observed at lower temperatures (Fig.5) could be related to the $T_{g}$ of the plasticized matrix and the peak at higher temperatures should correspond to the $T_{g}$ of the dried matrix. The Fox relationship is commonly used to describe the plasticizing effect:

$$
\frac{1}{T_{g P}}=\frac{w_{1}}{T_{g 1}}+\frac{w_{2}}{T_{g 2}}
$$


being $T_{g 1}, T_{g 2}$ and $T_{g P}$ the $T_{g}$ of water, dry epoxy and plasticized epoxy respectively, and $w_{1}=1-w_{2}$ the water weight fraction. From the $T_{g}$ of water $T_{g 1}=100-150 \mathrm{~K}$ [32] $T_{g 2}$ of the unaged epoxy given on Table 3, and the mass of absorbed water corresponding to two years of immersion (see Fig 2) $w_{1} \sim 0.019-0.018$, values of $T_{g} \sim 412-423 \mathrm{~K}$ (139$150^{\circ} \mathrm{C}$ ) are predicted for hydrothermally aged epoxy, which are in accordance with the position of the shoulder in $\tan \delta$ peak, located $20-26{ }^{\circ} \mathrm{C}$ lower than $\tan \delta$ peak.

It is well known that the addition of rigid nano-fillers increases the modulus of nanocomposites, as we have reported for samples similar to those studied here [6]. Fig. 6 shows the variation of $E^{\prime}$ with the temperature for one year hydrothermally aged samples, it can be seen that the $E^{\prime}$-temperature curves of the nanocomposites are above those corresponding to the neat epoxy thermoset, evidencing that fillers increase $E^{\prime}$ in both rubbery $\left(T>T_{g}\right)$ and glassy region $\left(T<T_{g}\right)$. Fig. 7 compares the $E^{\prime}$ modulus of aged and unaged samples in the glassy region (at $60^{\circ} \mathrm{C}<T_{g}$ ) for neat epoxy and nanocomposites. GNPs and CNTs cause an increase of $E^{\prime}$ in the glassy region $\left(E_{G}^{\prime}\right)$ and this effect appears both in the unaged and water aged samples. Namely $16 \%$ increment of $E_{G}^{\prime}$ is observed for $0.1 \mathrm{C}$ nanocomposite, and $25 \%, 33 \%, 37 \%$ and $48 \%$ for $5 \mathrm{G}-0.1 \mathrm{C}$, 8G-0.1C, 10G-0.1C and 12G-0.1C hybrid nanocomposites respectively. Hydrothermal ageing provokes a noticeable reduction in the glassy modulus this decrease could be attributed to water plasticization of the matrix and to degradation of the polymer-filler interface. As the $E_{G}^{\prime}$ decrease is about $15 \%$ regardless of whether it is referred to neat epoxy or nanocomposites, the dominant factor should be the water plasticization of the matrix.

On the other hand, as can be seen in Fig. 6 in the rubbery region the increments of $E_{R}^{\prime}$ due to the addition of 0.1CNTs is almost unnoticeable (6\%), however the addition of GNPs provokes significant increments of $E_{R}^{\prime}$, that is, $75 \%, 100 \%, 130 \%$ and $130 \%$ for 5G-0.1C, 8G-0.1C, 10G-0.1C and 12G-0.1C hybrid nanocomposites respectively. There was not detected any decrease of modulus, $E_{R}^{\prime}$, after hydrothermal ageing as it is show in Fig. 8 that compares $E_{R}^{\prime}$ of unaged and water aged samples at $T=200^{\circ} \mathrm{C}>T_{g}$ for neat epoxy and nanocomposites. The rubbery modulus of neat epoxy is correlated with its crosslink degree, thus the similar values of $E^{\prime}{ }_{R}$ obtained for aged and unaged epoxy samples indicate that there is no degradation of the epoxy thermoset. Moreover, for nanocomposites both unaged and water aged samples behave similar in the elastomeric zone, this is the consequence of drying the samples during the DMTA measurement, thus at $200^{\circ} \mathrm{C}$ the absorbed water has been eliminated. Therefore there is no evidence of 
irreversible degradation caused by water absorption, which agrees with the morphological study.

\subsection{Effect of hydrothermal ageing on the electrical conductivity of nanocomposites}

The effect of hydrothermal ageing on electrical conductivity was measured at room temperature $\left(24^{\circ} \mathrm{C}\right)$ using samples that were previously aged in water at $40^{\circ} \mathrm{C}$. The water absorbed for these samples corresponds to $2500 \mathrm{~s}^{1 / 2} \cdot \mathrm{mm}^{-1}$ in Fig. 2. The electrical conductivity for unaged and hydrothermally aged specimens is shown in Fig. 9. It is well known that graphitic fillers give an increment of the electrical conductivity of the epoxy resin. As we have reported in a previous study [6], the addition of $0.1 \%$ CNT induces an important increase of electrical conductivity, since the CNT load is higher than the percolation threshold. However, the addition of amino functionalized GNPs up to $12 \%$ renders a very low value of the electrical conductivity, this is because of the functionalization of GNPs that induces reduction of the intrinsic electrical conductivity of the graphene nanoplatelets, moreover the reaction of amine groups of GNPs with the epoxy groups of the matrix hinders the electrical contact between graphene nanoplatelets lowering the electrical conductivity.

Few published results deal with electrical properties of epoxy hybrid nanocomposites by combining CNTs and GNPs. Lee et al. [10] have reported the same surface conductivity for CNTs and CNT-GNPs nanocomposites. Yue et al. [11] have found that the CNT-GNP combination reduces the percolation threshold. Li et al. [12] have reported synergy in the electrical conductivity of a CNT-GNP nanocomposite. Safdari et al. [13] have reported both, high decrease in the electrical percolation threshold and synergy effect for the electrical conductivity for nanocomposites. As it is shown in Fig.9 the addition of $0.1 \%$ CNT to GNPs-based nanocomposites increases their electrical conductivity 3 orders of magnitude, but no synergistic effect is observed as neither of the hybrid compositions overcomes the $0.1 \mathrm{C}$ nanocomposite conductivity. This would be a consequence of the reduced conductivity of functionalized GNPs, that don't add efficient conductive pathways in the hybrid nanocomposite. Regarding to the effect of hydrothermal ageing, it is remarkable that water aged specimens do not show any deterioration of electrical conductivity, as it is shown in Fig. 9 aged and unaged nanocomposites have similar electrical conductivity, this is another indication of no degradation of the epoxy matrix neither of the electrical network by water. 


\section{Conclusions}

The water absorption and its effect on the thermo-mechanical properties of hybrid CNT/ GNP/epoxy nanocomposites were studied. The content of CNTs was $0.1 \mathrm{wt} \%$ which gives percolation referred to electrical conductivity. The GNPs used were amino fuctionalized in order to enhance the interface nanofiller-epoxy matrix. The GNPs content ranged from 5 to $12 \mathrm{wt} \%$. These nanocomposites were selected because they exhibit a synergetic effect on the stiffness.

The isothermal water absorption was measured until very long times (almost two years). Nano-fillers in the epoxy matrix led to a significant reduction of the water absorption. The water absorption curves were fitted to a two-step diffusion model, the first step is diffusion controlled and the second one is associated with relaxation of the epoxy matrix. The diffusion coefficients are lower in the nanocomposites than in neat epoxy thermoset, due to the tortuous paths created by the addition of the nano-fillers.

$T_{g}$ of nanocomposites is higher than the $T_{g}$ of neat epoxy and is similar in aged and unaged samples, evidencing that no chemical degradation took place. CNTs and GNPs improve the rigidity of the nanocomposites both before and after water ageing.

Whatever the temperature the storage modulus shows a continuous increase with the increase of the nanoparticles content, the effect of hydrothermal ageing is to decrease the modulus only in the glassy state $\left(T<T_{g}\right)$.

The addition of $0.1 \%$ CNTs to amine functionalized GNPs-based nanocomposites increases their electrical conductivity 3 orders of magnitude. Water aged specimens show similar electrical conductivity than unaged ones, which is another indication of no degradation of the samples by water. These results together with the morphology observed by FEG-SEM allow to affirm that no significant degradation takes place over two years of immersion in water at $40{ }^{\circ} \mathrm{C}$ in the epoxy thermoset neither in the hybrid nanocomposites.

\section{Acknowledgement}

The authors would like to acknowledge funding from the Ministry of Economy and Competitiveness of Spain: Project MAT2016-78825. 


\section{References}

[1] S.Y. Yang, W. N. Lin, Y.L. Huang, H. W. Tien, J. Wang, C.M. Ma, S. Li, J.Y. Wang, Synergetic effects of graphene platelets and carbon nanotubes on the mechanical and thermal properties of epoxy composites, Carbon 49 (2011) 793-803.

[2] P. C. Ma, N. A. Siddiqui, G. Marom, J. K. Kim, Dispersion and functionalization of carbon nanotubes for polymer-based nanocomposites: A review, Composites: Part A 41 (2010) 1345-1367.

[3] T. Kuillaa, S. Bhadra, D. Yaoa, N. H. Kim, S. Bose, J. H. Lee. Recent advances in graphene based polymer composites, Prog. Polym. Sci. 35 (2010) 1350-1375

[4] N. P. Singh, V.K. Gupta, A. P. Singh, Graphene and carbon nanotube reinforced epoxy nanocomposites: A review. Polymer 180 (2019)121724

[5] A. S. Patole, S.t P. Patole, S. Jung, J. Yoo, J. An, T. Kim, Self assembled graphene/carbon nanotube/polystyrene hybrid nanocomposite by in situ microemulsion polymerization, Eur. Polym. J. 48 (2012) 252-259.

[6] S. G. Prolongo, R. Moriche, A. Ureña, S. Florez, I. Gaztelumendi, C. Arribas, M. G. Prolongo, Carbon nanotubes and graphene into thermosetting composites: Synergy and combined effect, J. Appl. Polym. Sci.135 (2018) 46475-46482.

[7] S. Chatterjee, F. Nafezarefi, N.H. Tai, L. Schlagenhauf, F.A. Nüesch, B.T.T. Chu, Size and synergy effects of nanofiller hybrids including graphene nanoplatelets and carbon nanotubes in mechanical properties of epoxy composites, Carbon 50 (2012) 5380-5386.

[8] A.A. Moosa, A. Ramazani S.A., F.A.K. Kubba, M. Raad, Synergetic effects of graphene and nanofunctionalized carbon nanotubes hybrid reinforced epoxy matrix on mechanical thermal and wettability properties of nanocomposites, Am. J. Mater. Sci.7 (2017) 1-11.

[9] W. Li, A. Dichiara, J. Bai, Carbon nanotube-graphene nanoplatelet hybrids as high performance multifunctional reinforcements in epoxy composites, Compos. Sci. Technol. 74 (2013) 221-227.

[10]Y.S. Lee, Y.H. Park, K.H. Yoon, Flexural, electrical, thermal and electromagnetic interference shielding properties of $\mathrm{xGnP}$ and carbon nanotube filled epoxy hybrid nanocomposites, Carbon Letters 24 (2017) 41-46.

[11] L.Yue, G. Pircheraghi, S. A. Monemian, I. Manas-Zloczower. Epoxy composites with carbon nanotubes and graphene nanoplatelets. Dispersion and synergy effects, Carbon78 (2014) 268-278. 
[12] J. Li, P.S. Wong, J.K. Kim, Hybrid nanocomposites containing carbon nanotubes and graphite nanoplatelets, Mater. Sci. Eng. A: Struct. Mater. 660 (2008) 483-484.

[13] M. Safdari, M. S. Al-Haik. Synergistic electrical and thermal transport properties of hybrid polymeric nanocomposites based on carbon nanotubes and graphite nanoplatelets, Carbon 64 (2013)11-121.

[14] K. Liu, S. Chen , Y. Luo , D. Jia , H. Gao , G. Hu, L. Liu, Edge-functionalized graphene as reinforcement of epoxy-based conductive composite for electrical interconnects, Compos. Sci. Tech. 88 (2013) 84-91.

[15] J. Kim, B. Yim, J. Kim, The effects of functionalized graphene nanosheets on the thermal and mechanical properties of epoxy composites for anisotropic conductive adhesives (ACAs). Microelectron. Reliab. 52 (2012) 595-602.

[16] B. Ahmadi-Moghadam, M. Sharafimasooleh, S. Shadlou, F. Taheri, Effect of functionalization of graphene nanoplatelets on the mechanical response of graphene/epoxy composites, Mater. Design 66 (2015) 142-149.

[17] S.G. Prolongo, R. Moriche, A. Jiménez-Suárez, M. Sánchez, A. Ureña, Advantages and disadvantages of the addition of graphene nanoplatelets to epoxy resins. Eur. Polym. J. 61 (2014) 206-214.

[18] S.G. Prolongo, A. Jimenez-Suarez, R. Moriche, A. Ureña. In situ processing of epoxy composites reinforced with graphene nanoplatelets. Compos. Sci. Technol. 86 (2013) 185-191.

[19] Z. A. Ghaleb, M. Jaafar, Z. M. Ariff, Effect of amino-functionalized on tensile and electrical properties of graphene nanopowder filled epoxy thin film nanocomposite, J. Polym Mater. 33 (2016) 155-164.

[20] Z. A. Ghaleb, M. Mariatti, Z. M. Ariff, J. Ervina, Preparation and properties of amine functionalized graphene filled epoxy thin film nanocomposites for electrically conductive adhesive, J. Mater. Sci.: Mater. Electron. 29 (2018) 3160-3169

[21] S. Alessi, D. Conduruta, G. Pitarresi, C. Dispenza, G. Spadaro, Accelerated ageing due to moisture absorption of thermally cured epoxy resin/polyethersulphone blends. Thermal, mechanical and morphological behavior, Polym. Degrad. Stabil. 96 (2011) 642-648.

[22] Y.C. Lin, X. Chen, Moisture sorption-desorption-resorption characteristics and its effect on the mechanical behaviour of the epoxy system, Polymer 46 (2005) 1199412003. 
[23] O. Starkova, S. Chandrasekaran, T. Schnoor, J. Sevcenko, K. Schulte, Anomalous water diffusion in epoxy/carbon nanoparticle composites, Polym. Degrad. Stabil. 164 (2019) 127-135.

[24] J.M. Tomasi, I.D. Helman, W.A. Pisani, D.R. Klimek-McDonald, S. Chinkanjanarot, I. Miskioglu, J.A. King, G.M. Odegard, Accelerated hydrothermal aging of cycloaliphatic epoxy/graphene nanoparticle composites, Polym. Degrad. Stabil. 133 (2016) 131-135.

[25] C. Bockenheimer, D. Fata, W. Possart, New aspects of aging in epoxy networks. II. Hydrothermal aging, J. Appl. Polym. Sci. 91 (2004) 369-377.

[26] J. Zhou, J.P. Lucas, Hygrothermal effects of epoxy resin. Part I: the nature of water in epoxy, Polymer 40 (1999) 5513-5522.

[27] J. Zhou, J. P. Lucas, Hygrothermal effects of epoxy resin. Part II: variations of glass transition temperature, Polymer 40 (1999) 5513-5522.

[28] O. Starkova, S. Chandrasekaran, L.A.S.A. Prado, F. Tölle, R. Mülhaupt, K. Schulte, Hydrothermally resistant thermally reduced graphene oxide and multi-wall carbon nanotube based epoxy nanocomposites, Polym. Degrad. Stabil. 98 (2013) 519526.

[29] H.K. Liu, Y.C. Wang, T.H. Huang, Moisture effect on mechanical properties of graphene/epoxy nanocomposites, J. Mech., 32, (2016) 673-682

[30] S. G. Prolongo, A. Jiménez-Suárez, R. Moriche, A. Ureña, Influence of thickness and lateral size of graphene nanoplatelets on water uptake in epoxy/graphene nanocomposites, Appl. Sci. 8 (2018)1550-1560.

[31] J.J. Crank. The Mathematics of Diffusion, Oxford Clarendon Press London (1975)

[32] C. Arribas, L. Sepúlveda, C. Salom, R.M. Masegosa, M.G. Prolongo, Morphology effect on the hydrothermal ageing of a thermoplastic modified epoxy thermoset, Polym. Eng. Sci. 47 (2007) 960-968.

[33] S.G. Prolongo, M.R. Gude, A. Ureña, Water uptake of epoxy composites reinforced with carbon nanofillers, Compos. Part A 43 (2012) 2169-2175.

[34] A.F. Abdelkader, J.R. White, Water absorption in epoxy resins: the effects of the crosslinking agent and curing temperature, J. Appl. Polym. Sci. 98 (2005) 2544-2549.

[35]. L.R. Bao, A.F. Yee, C.Y.C. Lee, Moisture absorption and hygrothermal aging in a bismaleimide resin, Polymer 42 (2001) 7327-7333. 
[36] L.R. Bao, A.F. Yee, Moisture diffusion and hygrothermal aging in bismaleimide matrix carbon fiber composites-part I: uni-weave composites, Compos. Sci. Technol. 62 (2002) 2099-2110.

[37] V.M. Karbhari, G. Xian, Hygrothermal effects on high VF pultruded unidirectional carbon/epoxy composites: moisture uptake, Compos. B Eng. 40 (2009) 41-49.

[38] Z. Lu, G. Xian, H. Li, Effects of thermal aging on the water uptake behavior of pultruded BFRP plates, Polym. Degrad. Stab. 110 (2014) 216-224.

[39] M. Di Filippo, S. Alessi, G. Pitarresi, M.A. Sabatino, A. Zucchelli, C.Dispenza, Hydrothermal aging of carbon reinforced epoxy laminates with nanofibrous mats as toughening interlayers, Polym. Degrad. Stabil. 126 (2016) 188-195.

[40] B. Tan, N.L.Thomas, A review of the water barrier properties of polymer/clay and polymer/graphene nanocomposites. J. Membrane Sci. 514 (2016) 595-612.

[41] Q. Yang, G. Xiang, V.M. Karbhari, Hygrothermal ageing of an epoxy adhesive used in FRP strengthening of concrete, J. Appl. Polym. Sci. 104 (2007) 2607-2617.

[42] V. M. Karbhari, G. Xian, Hygrothermal effects on high $V_{F}$ pultruded unidirectional carbon /epoxy composites: moisture uptake, Composites: Part B 40 (2009) 41-49.

[43] Xian G, Karbhari VM. Segmental relaxation of water-aged ambient cured epoxy. Polymer Degradation and Stability 92 (2007)1650-1659. 
Table 1. Composition of the studied samples.

\begin{tabular}{|ccccc|}
\hline Sample & Name & $\begin{array}{c}\text { GNP } \\
\mathbf{( w t} \%)\end{array}$ & $\begin{array}{c}\text { CNT } \\
(\mathbf{w t} \%)\end{array}$ & $\begin{array}{c}\text { Total nanofiller } \\
(\mathbf{w t} \%)\end{array}$ \\
\hline Neat epoxy & Epoxy & 0 & 0 & 0 \\
\hline CNT/epoxy & $0.1 \mathrm{C}$ & 0 & 0.1 & 0.1 \\
\hline GNP/epoxy & $12 \mathrm{G}$ & 12 & 0 & 12.0 \\
\hline $\begin{array}{c}\text { Hybrid } \\
\text { GNP/CNT/epoxy }\end{array}$ & 5G-0.1C & 5 & 0.1 & 5.1 \\
& 10G-0.1C & 10 & 0.1 & 10.1 \\
& 12G-0.1C & 12 & 0.1 & 12.1 \\
\hline
\end{tabular}


Table 2. Quasi-equilibrium water uptake values $\left(M_{\infty 0}\right)$ diffusion coefficients $(D)$ and values of the parameter of the second stage $(k)$, according to Eq. (3).

\begin{tabular}{|cccc|}
\hline Material & $\begin{array}{c}\boldsymbol{M}_{\infty \mathbf{0}} \\
(\%)\end{array}$ & $\begin{array}{c}\boldsymbol{D} \cdot \mathbf{1 0}^{7} \\
\left(\mathrm{~mm}^{2} \cdot \mathrm{s}^{-1}\right)\end{array}$ & $\begin{array}{c}\boldsymbol{k} \cdot \mathbf{1 0}^{5} \\
\mathrm{~s}^{-1 / 2}\end{array}$ \\
\hline Epoxy & $1.50 \pm 0.01$ & $6.45 \pm 0.06$ & $5.2 \pm 0.2$ \\
0.1C & $1.40 \pm 0.01$ & $6.28 \pm 0.04$ & $4.9 \pm 0.1$ \\
12G & $1.30 \pm 0.01$ & $5.34 \pm 0.10$ & $4.1 \pm 0.3$ \\
5G-0.1C & $1.36 \pm 0.01$ & $5.95 \pm 0.01$ & $4.5 \pm 0.3$ \\
8G-0.1C & $1.36 \pm 0.01$ & $5.87 \pm 0.10$ & $4.1 \pm 0.3$ \\
10G-0.1C & $1.34 \pm 0.01$ & $5.29 \pm 0.06$ & $4.0 \pm 0.3$ \\
12G-0.1C & $1.33 \pm 0.01$ & $5.19 \pm 0.02$ & $4.0 \pm 0.2$ \\
\hline
\end{tabular}

Table 3. Temperature values of $\tan \delta_{\max }$ ( $T_{g}$ from DMTA) for unaged and hydrothermally aged specimens at $40^{\circ} \mathrm{C}$ after 1 year and after almost 2 years (values in brackets)

\begin{tabular}{|c|c|c|}
\hline \multirow{2}{*}{ Material } & \multicolumn{2}{|c|}{$\left.\boldsymbol{T}_{\boldsymbol{g}} \mathbf{}^{\mathbf{}} \mathbf{C}\right)$} \\
\cline { 2 - 3 } & Unaged & $\begin{array}{c}\text { Hydrothermally } \\
\text { aged }\end{array}$ \\
\hline Epoxy & 165 & $167(168)$ \\
\hline 0.1C & 169 & $169(168)$ \\
\hline 12G & 171 & $169(169)$ \\
\hline 5G-0.1C & 170 & $171(169)$ \\
\hline 8G-0.1C & 171 & $172(172)$ \\
\hline 10G-0.1C & 171 & $170(171)$ \\
\hline 12G-0.1C & 174 & $174(174)$ \\
\hline
\end{tabular}



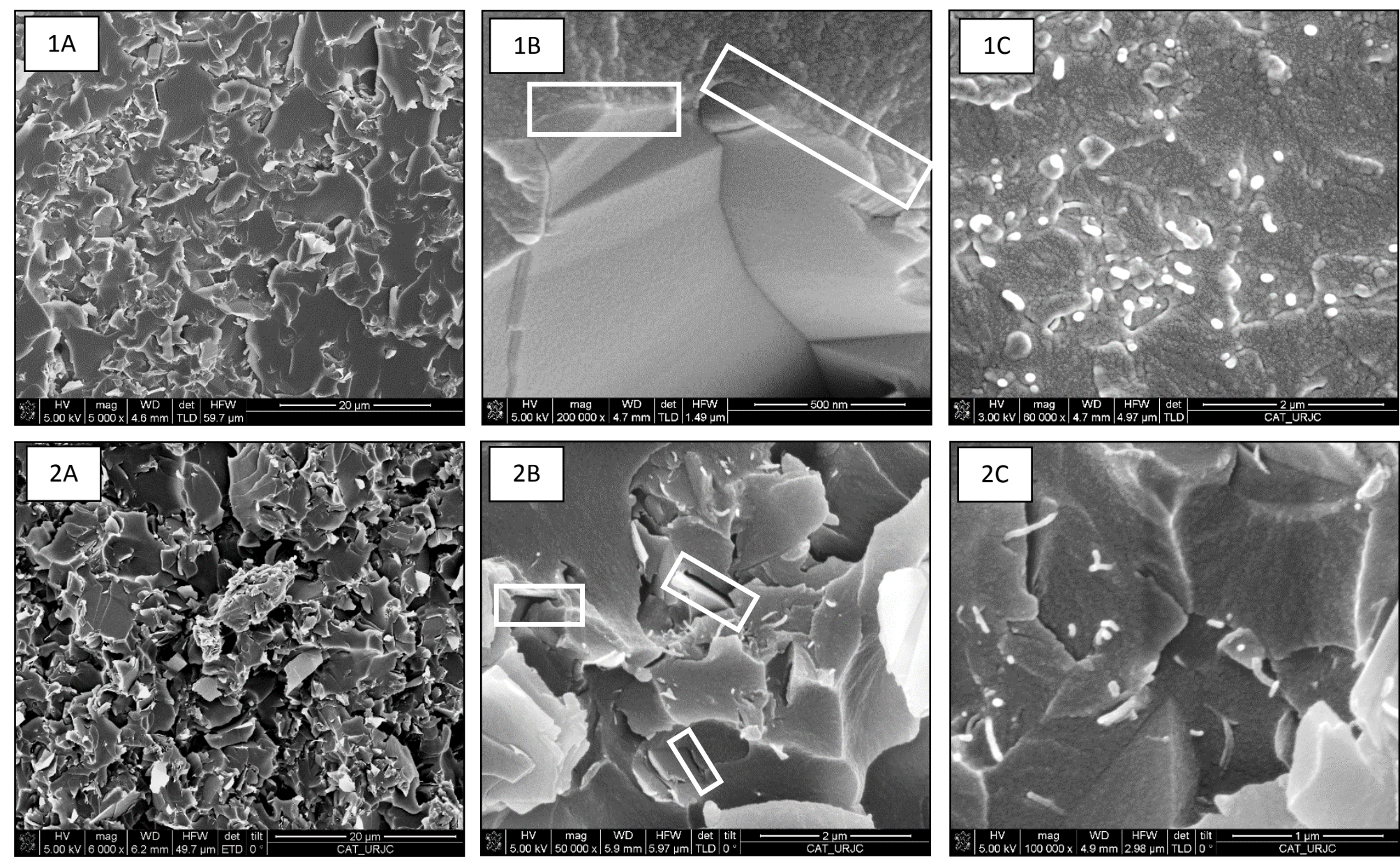

Fig. 1. FEG-SEM micrographs of 8G-0.1C nanocomposite: unaged samples (1A, 1B and $1 \mathrm{C})$ and water aged $\left(1\right.$ year $\left.40^{\circ} \mathrm{C}\right)$ samples $(2 \mathrm{~A}, 2 \mathrm{~B}$ and $2 \mathrm{C})$ at different magnifications 


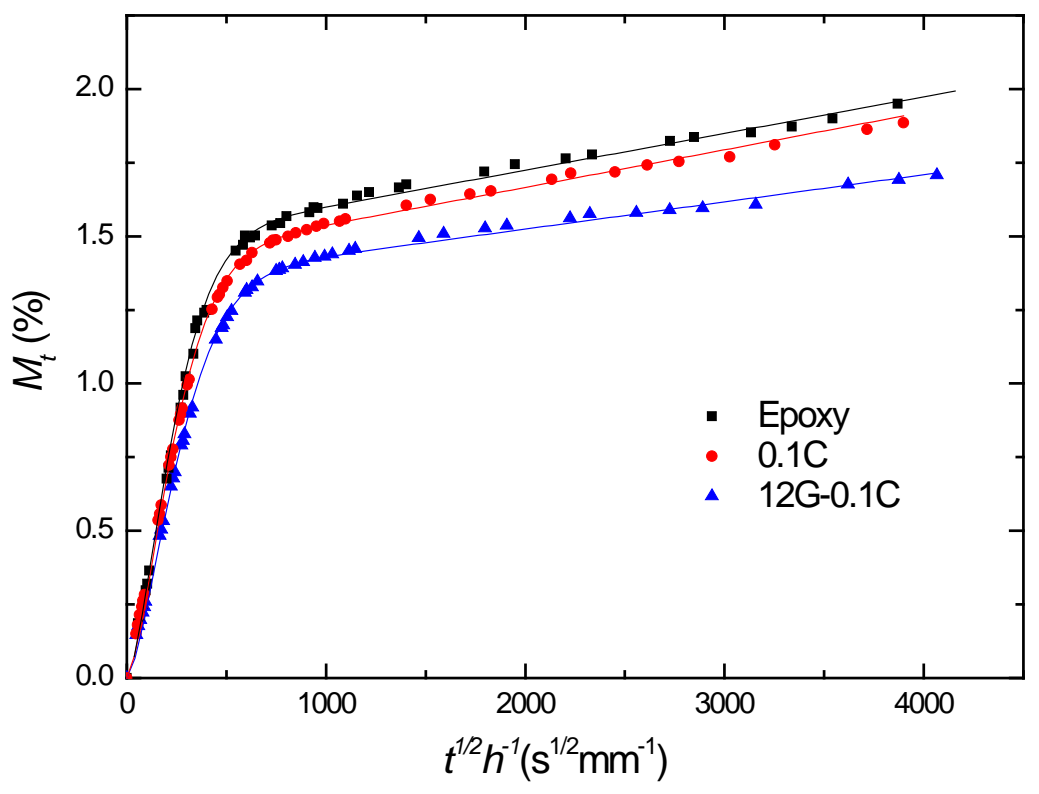

Fig. 2. Water absorption curves for neat epoxy ( $\mathbf{})$ and nanocomposites: $0.1 \mathrm{C}(\bullet)$ and 12G-0.1C ( $\boldsymbol{\Delta})$, lines represent the theoretical fitting (Eq.3). 


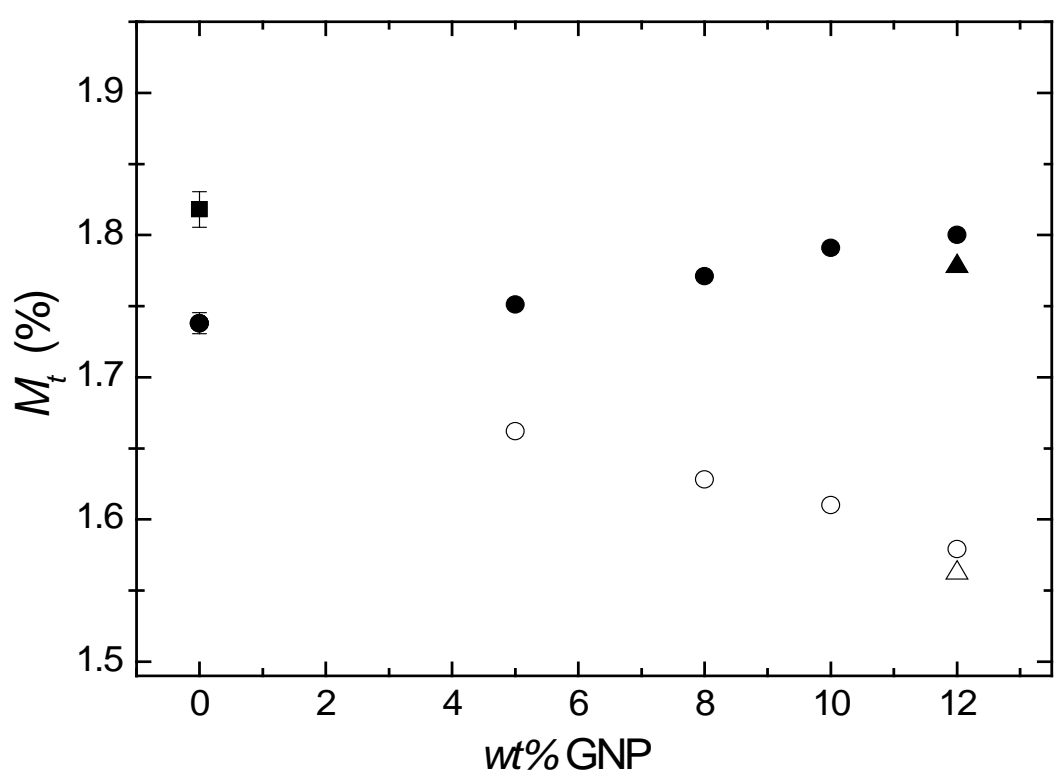

Fig. 3. Water absorption at $2500 \mathrm{~s}^{1 / 2} \cdot \mathrm{mm}^{-1}$ as a function of GNP content: referred to the total mass of the specimen (open symbols) and referred to the epoxy mass (filled symbols) for neat epoxy: $(\boldsymbol{\bullet}), 12 \mathrm{G}$ nanocomposite $(\boldsymbol{\Delta}, \Delta)$ and hybrid graphene-0.1\%CNT nanocomposites $(\bullet \circ)$. When the error bars are smaller than the size of the symbol they are not shown. 


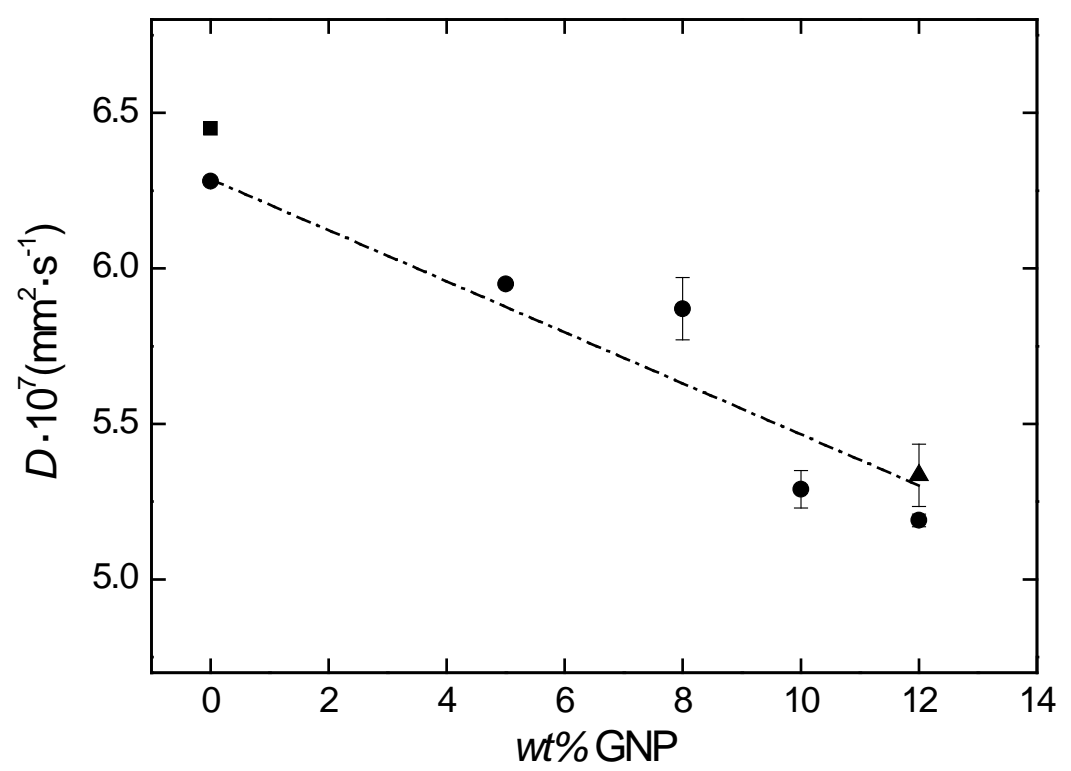

Fig.4. Diffusion coefficients for: neat epoxy $(\boldsymbol{\square}), 12 \mathrm{G}$ nanocomposite $(\boldsymbol{\Delta})$ and hybrid graphene-0.1\%CNT nanocomposites $(\bullet)$. When the error bars are smaller than the size of the symbol they are not shown. 

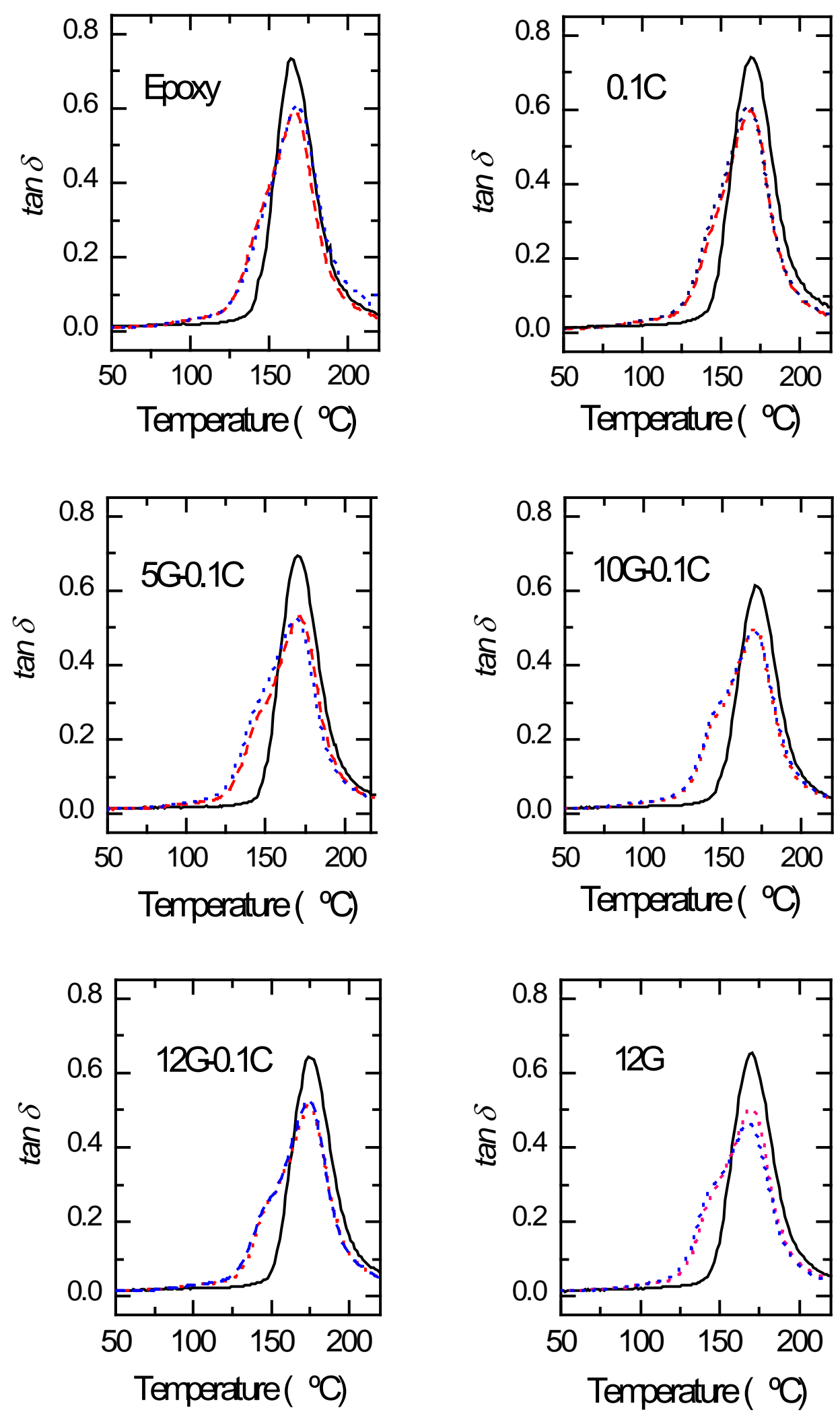

Fig. 5. DMTA tan $\delta$ - temperature curves for samples: unaged (-), one year aged ( $\cdots)$ and two year aged (---). 


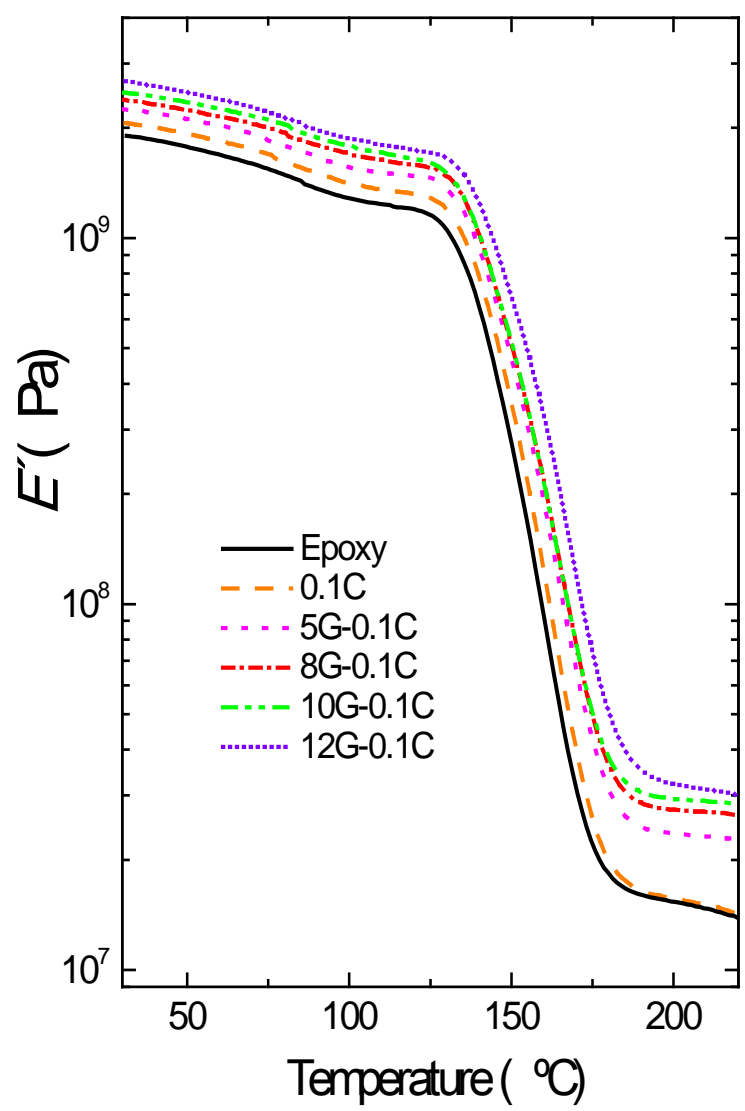

Fig. 6. DMTA $E^{\prime}$-temperature curves for one year aged specimens.

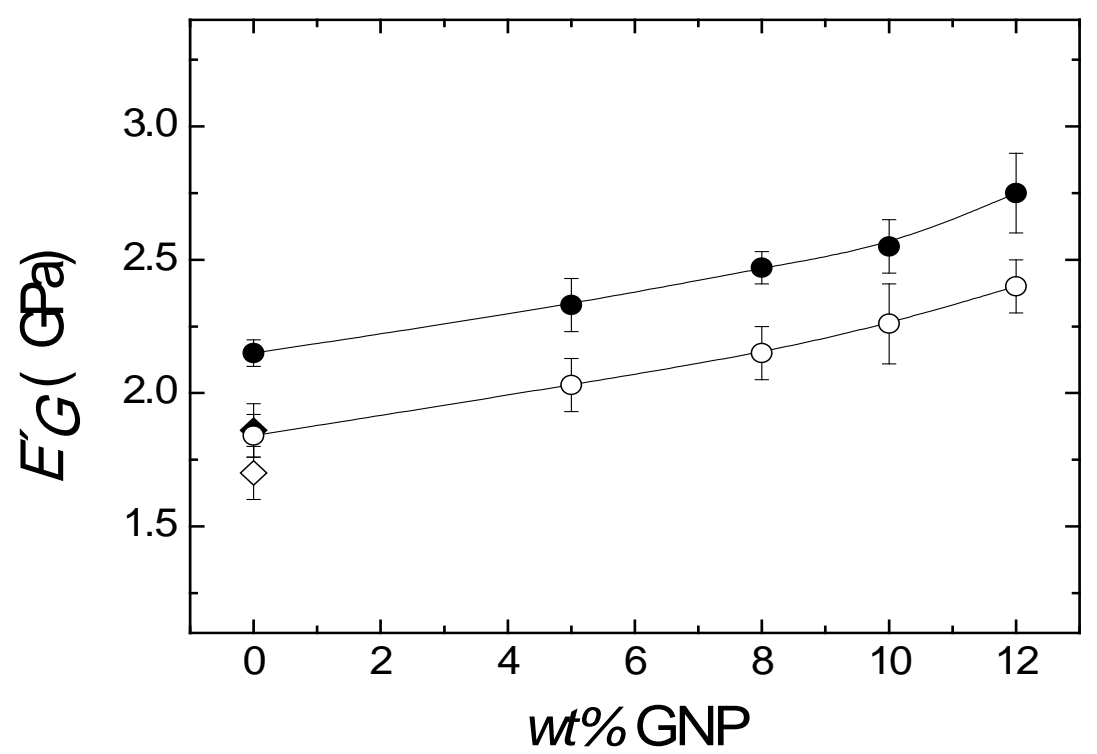

Fig. 7. $E^{\prime}$ in the glassy state $\left(60^{\circ} \mathrm{C}\right)$ for neat epoxy $(\diamond)$ and hybrid graphene-0.1\%CNT nanocomposites $(\bullet)$. Filled symbols stand for unaged samples and open symbols for aged samples. 


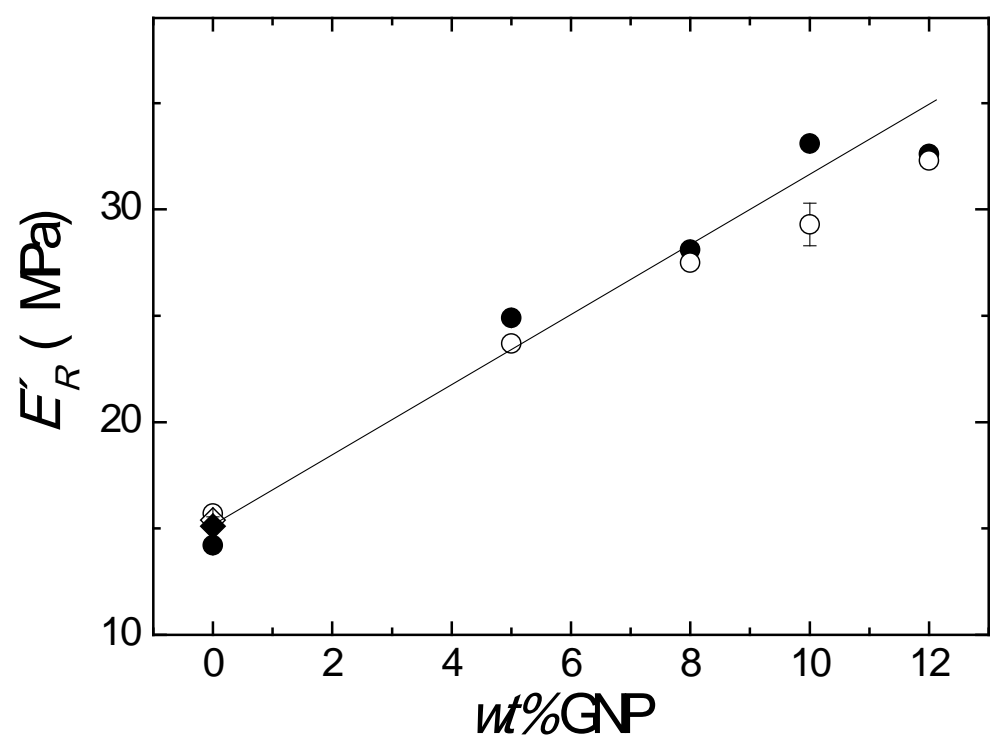

Fig. 8. $E^{\prime}$ in the rubbery state $\left(200^{\circ} \mathrm{C}\right)$ for neat epoxy $(\diamond)$ and hybrid graphene- $0.1 \% \mathrm{CNT}$ nanocomposites $(\bullet)$. Filled symbols stand for unaged samples and open symbols for aged samples. When the error bars are smaller than the size of the symbol they are not shown.

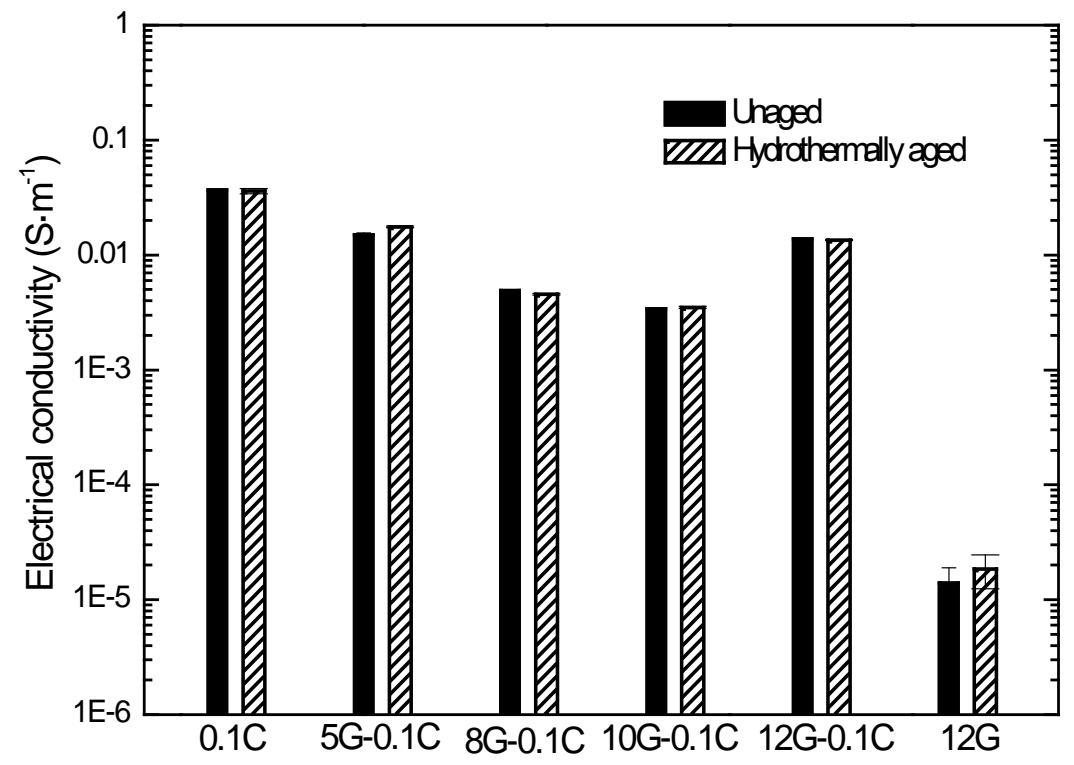

Fig. 9. Electrical conductivity for the nanocomposites: $0.1 \mathrm{C}, 12 \mathrm{G}$ and hybrid graphene-CNT nanocomposites: 5G-0.1C, 8G-0.1C 10G-0.1C and 12G-0.1C. 